\title{
Determination of Optimal Opening Scheme for Electromagnetic Loop Networks Based on Fuzzy Analytic Hierarchy Process
}

\author{
Yang Li, ${ }^{1}$ Yahui Li, ${ }^{1}$ Zijiao Han, ${ }^{2}$ Guoqing Li, ${ }^{1}$ Kai Gao, ${ }^{2}$ and Zhenhao Wang \\ ${ }^{1}$ School of Electrical Engineering, Northeast Dianli University, Jilin 132012, China \\ ${ }^{2}$ Liaoning Electric Power Company Limited, Shenyang 110006, China \\ Correspondence should be addressed to Yang Li; 876464104@qq.com
}

Received 19 February 2016; Revised 23 May 2016; Accepted 14 June 2016

Academic Editor: Thomas Hanne

Copyright (c) 2016 Yang Li et al. This is an open access article distributed under the Creative Commons Attribution License, which permits unrestricted use, distribution, and reproduction in any medium, provided the original work is properly cited.

\begin{abstract}
Studying optimization and decision for opening electromagnetic loop networks plays an important role in planning and operation of power grids. First, the basic principle of fuzzy analytic hierarchy process (FAHP) is introduced, and then an improved FAHPbased scheme evaluation method is proposed for decoupling electromagnetic loop networks based on a set of indicators reflecting the performance of the candidate schemes. The proposed method combines the advantages of analytic hierarchy process (AHP) and fuzzy comprehensive evaluation. On the one hand, AHP effectively combines qualitative and quantitative analysis to ensure the rationality of the evaluation model; on the other hand, the judgment matrix and qualitative indicators are expressed with trapezoidal fuzzy numbers to make decision-making more realistic. The effectiveness of the proposed method is validated by the application results on the real power system of Liaoning province of China.
\end{abstract}

\section{Introduction}

Scheme evaluations for opening electromagnetic loop networks provide an important reference for the planning and construction of power grids [1]. In recent years, with the rapid development of power grids, the problem of electromagnetic loop networks is becoming more and more serious in China. The transmission power of urban power networks grows rapidly with the development of power systems. In this process, China's power grids have two typical characteristics. On the one hand, the problem of short-circuit current (SCC) superscalar is serious; on the other hand, there are a lot of electromagnetic loops, which lead to the limitations of power transmission capacity.

In the early stage of high voltage power grid development, the power flow of high-low loop network is little. In this case, the electromagnetic loop networks can improve the reliability and flexibility of power grids [2]. But with the development of high-level voltage grid, the transmission load is increasing, and the electromagnetic loops become serious potential hazards [3]. The main hidden danger of the electromagnetic loop network is that large amounts of power flow pour into the lower voltage grid when the higher voltage grid is cut off, which can easily cause serious problems threatening the safe and stable operation of power systems. This operation mode also leads to a lot of potential problems in security and stability for power grids, such as overload, the difficulty of controlling power flow, SCC superscalar issue, complexity of protection setting, and even the destruction of the system thermal stability. Therefore, the operation mode of electromagnetic loop networks not only increases the difficulty of power grid operation and management, but also brings more uncertainty and more potential serious accidents [1].

An effective approach of eliminating the loop running is electromagnetic loop rejection to achieve the partitioning operation of power networks. Because of the complexity and diversity of power system operation, multifaceted calculation and analysis must be carried out before breaking up electromagnetic loop, comprising power flow calculation, stability calculation, SCC calculation, and network loss calculation. Moreover, other issues affecting the system operation should also be comprehensively considered to make reasonable and feasible decisions. Therefore, it is an important task to determine the optimal scheme for decoupling electromagnetic 
loop networks for the planning and construction of power grids.

Some beneficial explorations have been carried out on the issue of electromagnetic loop networks [4-9]. An approach based on group decision-making theory is proposed for accessing the different operation modes of electromagnetic loop network in [4]. A flexible looped network controller based on voltage source converter-high voltage direct current (VSC-HVDC) technology is used to solve the problems resulting from electromagnetic loop networks in [5]. In view of this operation mode, power flow control schemes for electromagnetic loop networks are studied in [6, 7]. A fuzzy comprehensive evaluation (FCE) of opening schemes is proposed for $500 \mathrm{kV}-220 \mathrm{kV}$ electromagnetic loop networks in [8]. In [9], analytic hierarchy process (AHP) is employed to solve the problem. Unfortunately, AHP is often influenced by subjective factors of different experts when determining the judgment matrix in different decision-making systems $[10,11]$. Meanwhile, FCE usually lacks systematicness and generality when it is used to solve different evaluation models [12]. Due to the complexity, ambiguity, and uncertainty of scheme evaluation, there is an urgent need for developing new approaches to solve this problem.

In the paper, a new multicriteria decision-making (MCDM) method based on fuzzy AHP (FAHP) for determination of the optimal scheme for decoupling electromagnetic loop networks is proposed. As a well-known MCDM method, AHP can effectively combine qualitative and quantitative analysis to ensure the rationality of the evaluation model, but it has some inherent disadvantages such as the abovementioned strong subjectivity, which restricts its further application in practice [13]. Fortunately, fuzzy set theory proposed by Zadeh in 1965 has proved to be an effective method to cope with such problems [14]. As the extension of AHP under fuzzy environments [15], FAHP has been successfully used for solving various multiattribute decisionmaking problems in the field of engineering [16-18]. In FAHP, the judgment matrix and qualitative indicators are expressed with trapezoidal fuzzy numbers to make decision-making more realistic and more rational [19-22]. The contribution of this paper can be divided into two aspects: on the one hand, how FAHP is applied to scheme evaluation for opening electromagnetic loop network in detail is demonstrated; on the other hand, significant performance improvements from applying the present approach to a real power system are further demonstrated.

The rest of this paper is arranged as follows. Section 2 introduces all the related concepts necessary to understand the proposal. Details of the present approach using FAHP are shown in Section 3. In Section 4, the proposed method is examined using a real test system, and finally the conclusions are drawn in Section 5.

\section{Related Concepts}

In this section, all the concepts necessary to understand the proposed approach are introduced. First of all, the definition of electromagnetic loop networks is given. Next, causes of electromagnetic loop networks are explained in brief. Then,

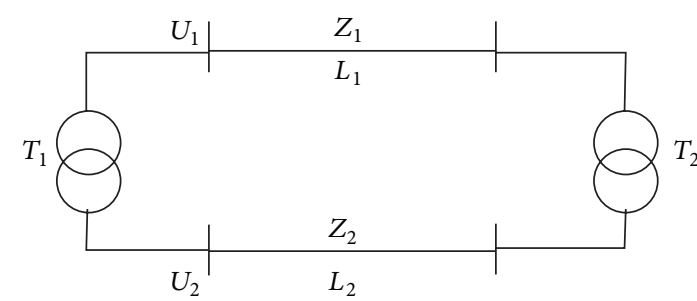

FIGURE 1: Schematic of electromagnetic loop networks.

the main problems existing in the operation mode of electromagnetic loop networks are listed. And finally, the basic principles and general steps of decoupling electromagnetic loop networks are described, respectively.

2.1. Definition of Electromagnetic Loop Networks. Electromagnetic loop refers to the lines with different voltage levels that are connected in parallel loop through the electromagnetic circuit of the connected transformers [6], as shown in Figure 1.

In Figure 1, the operating voltage $U_{1}$ of line $L_{1}$ is higher than the operating voltage $U_{2}$ of line $L_{2}, T_{1}$ and $T_{2}$ are transformers, and lines $L_{1}$ and $L_{2}$ constitute an electromagnetic loop through the magnetic circuits of $T_{1}$ and $T_{2}$.

2.2. Causes of Electromagnetic Loop Networks. Electromagnetic ring networks are the products of the process of power grid construction. When the high-level grid is not strong, there is certain rationality for its existence. But when high-level grid development is more mature, it should be considered to break up the electromagnetic ring network to ensure the hierarchical partitioning operation of power systems.

The main reasons why electromagnetic loop networks appear are described as follows:

(1) Some planning managers of power grids think that the use of electromagnetic loop network operation mode can improve the reliability of the local power supply, so that the power supply network can be enhanced, and construction investment in power networks can also be saved. They also think that the shortcomings of this electromagnetic ring are relatively minor.

(2) In the planning process, planners fail to conduct a reasonable forecasting and analysis for supply networks, which leads to the fact that some lines cannot meet the requirements for subsequent transmission capacity. To solve this problem, new higher levels of transmission lines are often added, eventually leading to the formation of electromagnetic ring network.

(3) In the operation and management of power grids, management is not concentrated enough due to the presence of multiple subsystems operating independently. Each subsystem makes construction of power grids in accordance with its own ideas and habits, which will inevitably lead to the parallel operation situation of different voltage levels grids. 
2.3. Problems Existing in the Operation Mode of Electromagnetic Loop Networks. The operation mode of electromagnetic loop networks brings troubles to the operation and management of power grids, and at the same time it also greatly increases uncertainties to the development of power grid. The main problems in this operation mode are as follows:

(1) Due to the dense power load, short transmission distance, and the integration of a lot of power supplies, this operation model will result in serious shortcircuit current superscalar problem.

(2) The operation mode of the whole power system is not unified because of the interaction of electromagnetic loop networks with different voltage grades. This makes it very difficult to control the power flows of the system.

(3) There are unreasonable distributions of power flow. It does not favour the economic operation of the power system, since the distributions of power flow cannot achieve the most economic levels.

(4) It might result in the serious security and stability problems for power system operation. When the lines in high voltage grades disconnect because of malfunction, the load-transfer problem will inevitably occur.

2.4. Basic Principles of Decoupling Electromagnetic Loop Networks. The basic principles of decoupling electromagnetic loop networks are as follows:

(1) The loop rejection should result in more reasonable flow distribution. Power flows should have more flexible controllability and be able to adapt to the changes in various operating modes. It should also ensure that no element overloads.

(2) The loop rejection should be able to ensure the security and stability of power systems. The stability indexes should be compared before and after breaking up the loops. If power flow transferring caused by disconnecting the high voltage lines seriously affected the stability of power systems, breaking up the electromagnetic loops is an urgent need.

(3) After breaking up the loops, the power networks should have a larger antijamming capability to meet the $\mathrm{N}-1$ requirements.

(4) After breaking up the loops, power supplies should be reasonably allocated among supply districts to provide sufficient reactive power compensation.

(5) After breaking up the loops, each partition should have sufficient connected aisles with reasonable distributions to timely transfer loads and avoid blackouts when an accident occurs.

(6) The short-circuit current usually decreases after breaking up the loops, and then it needs to determine what kind of line operation mode is most effective in reducing the short-circuit current.
(7) The network loss after breaking up the loops must be calculated and be compared with the network loss before breaking up the loops.

2.5. General Steps of Decoupling Electromagnetic Loop Networks. The general steps of decoupling electromagnetic loop networks are as follows:

(1) Operation Analysis of Electromagnetic Loop Networks. According to the current power network structure and the future planning of the network frame, analyze the existing situation and potential problems of electromagnetic loop networks under the typical operation modes of power systems.

(2) Determination of the Original Candidate Schemes. According to the above-mentioned basic decoupling principles, choose several original schemes to be evaluated through large amount of analysis and calculation, such as power flow calculation, SCC calculation, transient stability calculation, and static security analysis.

(3) Scheme Evaluation and Decision-Making. In this step, the main task is to determine the optimal opening scheme from the original candidate schemes through a comprehensive evaluation of all the candidate ones. It is the key in the whole process of decoupling the loop networks.

(4) Investment Estimation and Economic Comparison. Through comparison of the change of the system network loss and estimation of the required construction investment, comprehensively compare the economic benefits of decoupling the loop networks.

\section{Scheme Evaluations Based on FAHP}

In this section, the proposal is described as follows. First of all, the principle of FAHP is shown; in the second place, the improvement of FAHP is detailedly described; last but not the least, the evaluation model based on IFAHP is proposed.

3.1. Principle of FAHP. As a decision-making tool combining qualitative and quantitative analysis, AHP has been developed rapidly over the past decade. It is a decisionmaking method that refers to decomposing the relevant elements of the decision-making issue into the level of goals, guidelines, and programs. The whole process embodies the basic characteristics of the human decision-making, comprising decomposition, and judgment, comprehensive. However, AHP has some inherent shortcomings, such as the difficulty in checking and adjusting the consistency of the fuzzy judgment matrix.

The FAHP used in this paper is the extension of AHP under fuzzy environments, which employs AHP to build the hierarchical model, and replaces the pairwise comparison judgment matrix with a linguistic term set, such as "very unimportant, unimportant, little unimportant, generally, little important, important, very important" [23]. As shown in Table 1, the qualitative evaluations in the form of linguistic 
TABLE 1: Corresponding chart of qualitative evaluation and trapezium fuzzy numbers.

\begin{tabular}{lc}
\hline Qualitative attributes & Fuzzy numbers \\
\hline Very unimportant & $(0,0,0,0.2)$ \\
Little important & $(0.6,0.8,0.8,1)$ \\
Unimportant & $(0,0,0.1,0.3)$ \\
Important & $(0.7,0.9,1,1)$ \\
Little unimportant & $(0,0.2,0.2,0.4)$ \\
Very important & $(0.8,1,1,1)$ \\
Generally & $(0.3,0.5,0.5,0.7)$ \\
\hline
\end{tabular}

information can be transformed into quantitative indicators represented by trapezoidal fuzzy numbers by using bipolar scaling proposed by MacCrimmon and Wehrung [24].

On the one hand, FAHP inherits the advantages of AHP, which effectively combines qualitative analysis and quantitative analysis to ensure the rationality and systematicness of the evaluation model; on the other hand, FAHP fully takes advantage of the fuzzy nature of fuzzy set theory, which can handle experts' knowledge more objective. Therefore, this paper focuses on applications of FAHP to determine the optimal scheme for decoupling electromagnetic loop networks.

3.2. Improvement of FAHP. As previously mentioned, it is a problem in checking and adjusting the consistency of the fuzzy judgment matrix in AHP-based scheme evaluation methods including FAHP [25]. In this section, a scheme for improving the consistency of a fuzzy judgment matrix is proposed.

Suppose there are $n(n>2)$ attributes of decision-making problems denoted by $y_{1}, y_{2}, \ldots, y_{n}$; then, the attribute aggregation is $Y=\left\{y_{i} \mid i \in N\right\}, N=\{1,2, \ldots, n\}$.

Definition 1. Let $\mathbf{R}=\left(r_{i j}\right)_{n \times n}$ denote a trapezium fuzzy number complementary judgment matrix, if $r_{i j}=\left(a_{i j}, b_{i j}, c_{i j}, d_{i j}\right)$ is a trapezium fuzzy number, where $a_{i j} \leq b_{i j} \leq c_{i j} \leq d_{i j}, i, j \in N$, and the judgment matrix $\mathbf{R}$ satisfies

(I) $a_{i i}=b_{i i}=c_{i i}=d_{i i}=0.5, \forall i \in N$;

(II) $a_{i j}+d_{j i}=1, b_{i j}+c_{j i}=1, d_{i j}+a_{j i}=1, c_{i j}+b_{j i}=1 ; \forall i, j \epsilon$ $N, i \neq j$, where $r_{i j}$ describes the relative importance of $y_{i}$ compared to $y_{j}$.

The specific steps for improving the consistency of the judgment matrix are listed as follows.

Step 1. Suppose that the initial fuzzy judgment matrix $\mathbf{R}$ in the form of a linguistic term set and the critical value of the consistency index $\varepsilon$ are pregiven. Usually, $\varepsilon$ is set to 0.2 .

Step 2. Consistency check of the judgment matrix: the kernel of trapezium fuzzy number $r_{i j}$ is

$$
K\left(r_{i j}\right)= \begin{cases}a_{i j}, & \text { if } a_{i j}=b_{i j}=c_{i j}=d_{i j} \\ \frac{\left(c_{i j}\right)^{2}+\left(d_{i j}\right)^{2}-\left(a_{i j}\right)^{2}-\left(b_{i j}\right)^{2}+d_{i j} c_{i j}-a_{i j} b_{i j}}{3\left(c_{i j}+d_{i j}-a_{i j}-b_{i j}\right)}, & \text { otherwise, }\end{cases}
$$

where the corresponding kernel matrix $\widehat{\mathbf{R}}$ of the matrix $\mathbf{R}$ is $\widehat{\mathbf{R}}=\left(\widehat{r_{i j}}\right)_{n \times n}, \widehat{r_{i j}}=K\left(r_{i j}\right)$.

Based on the theorem proposed in [26], the necessary and sufficient condition that the kernel matrix $\widehat{\mathbf{R}}$ is an additive consistency of complementary judgment matrix is that the additive consistency index $\rho$ is equal to 0 , where $\rho$ is defined as

$$
\begin{aligned}
& \rho \\
& =\frac{2}{n(n-1)(n-2)} \sum_{i=1}^{n-1} \sum_{j=i+1}^{n} \sum_{\substack{k=1 \\
k \neq i, j}}^{n}\left|\widehat{r_{i j}}-\left(\widehat{r_{i k}}+\widehat{r_{k j}}-\frac{1}{2}\right)\right| .
\end{aligned}
$$

According to (2), calculate the consistency index $\rho$ of the kernel matrix $\widehat{\mathbf{R}}$. The greater the index $\rho$ is, the worse the consistency of the judgment matrix is. If $\rho<\varepsilon$, then it is considered that the consistency is met, and go to Step 7; else adjust the elements in the judgment matrix given by experts until the consistency is met.
Step 3. According to (3), calculate the priority weight vector $H=\left(H_{1}, H_{2}, \ldots, H_{n}\right)^{T}$,

$$
H_{i}=\frac{1}{n}-\frac{1}{2 \alpha}+\frac{1}{n \alpha} \sum_{k=1}^{n} \widehat{r_{i k}}
$$

where the parameter $\alpha \geq(n-1) / 2$, and then obtain the characteristic matrix $\mathbf{R}^{*}=\left(r_{i j}^{*}\right)_{n \times n}$

$$
r_{i j}^{*}=\alpha\left(H_{i}-H_{j}\right)+0.5 \text {. }
$$

Step 4. The deviation matrix $\mathbf{D}=\left(d_{i j}\right)_{n \times n}=\widehat{\mathbf{R}}-\mathbf{R}^{*}$ is calculated, and then $\left|d_{s t}\right|=\max \left\{\left|d_{i j}\right|: i<j, i, j \in n\right\}$ is determined.

Step 5. Update the matrix $\mathbf{R}$, and obtain the new fuzzy judgment matrix $\mathbf{R}^{\prime}=\left(r_{i j}^{\prime}\right)_{n \times n}$. If $d_{s t}>0$, then $r_{s t}$ and $r_{t s}$ are adjusted as follows: $r_{s t}^{\prime}=r_{s t}-\theta, r_{t s}^{\prime}=r_{t s}+\theta$; otherwise, $r_{s t}^{\prime}=r_{s t}+\theta, r_{t s}^{\prime}=r_{t s}-\theta$. Here, $\theta(0<\theta<1)$ is the adjustment quantity. 


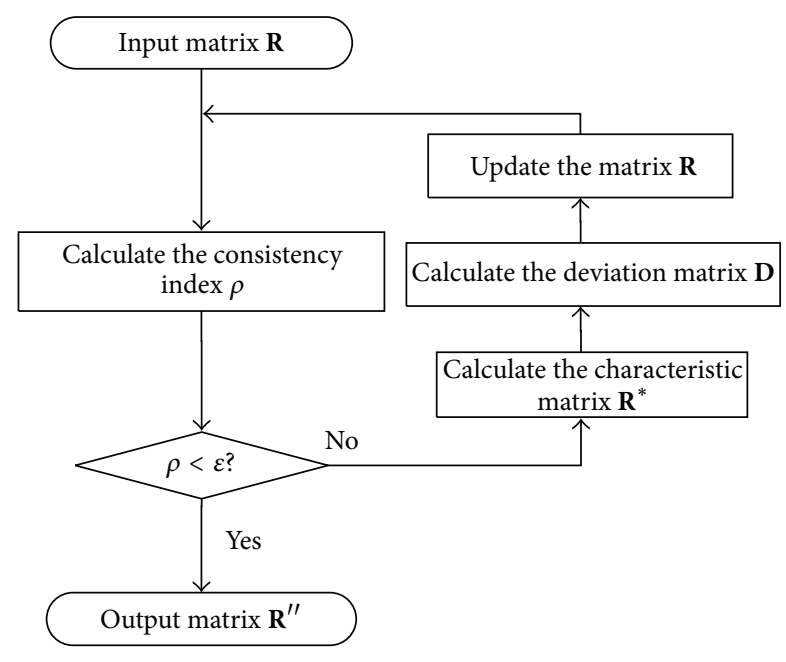

FIGURE 2: Flowchart of consistency improvement for FAHP.

Step 6. Repeat Step 2 to Step 5.

Step 7. Output the fuzzy judgment matrix $\mathbf{R}^{\prime \prime}$, which meets the consistency.

The flowchart of consistency improvement for FAHP is shown in Figure 2.

3.3. Evaluation Model Based on IFAHP. The proposed scheme evaluation method based on improved FAHP (IFAHP) mainly includes the following steps: establishment of the hierarchical structure model, construction of fuzzy judgment matrices, normalization of the indexes, and comprehensive ranking of the schemes, as shown in Figure 3.

3.3.1. Construction of the Hierarchy Model. According to the basic principles of decoupling electromagnetic loop networks, the hierarchical structure model is established, as shown in Figure 4.

In this hierarchy model, the goal layer $\mathbf{A}$ is the optimal scheme sequence; the criterion layer consists of three parts: the security criterion $\mathbf{B}_{1}$, the stability criterion $\mathbf{B}_{2}$, and the economy criterion $\mathbf{B}_{3}$; the index layer is made up of the $N-1$ index $\mathbf{C}_{1}$, the average rate of line overloads index $\mathbf{C}_{2}$, the average rate of transformer overloading index $\mathbf{C}_{3}$, the average three-phase short-circuit current index $\mathbf{C}_{4}$, the average single-phase short-circuit current index $\mathbf{C}_{5}$, the critical clearing time index $\mathbf{C}_{6}$, and the network loss index $\mathbf{C}_{7}$; the scheme layer consists of all the candidate schemes. The main indexes influencing the security criterion $\mathbf{B}_{1}$ are $\mathbf{C}_{1}, \mathbf{C}_{2}$, $\mathbf{C}_{3}, \mathbf{C}_{4}$, and $\mathbf{C}_{5}$; the main indexes influencing the stability criterion $\mathbf{B}_{2}$ are $\mathbf{C}_{2}, \mathbf{C}_{3}, \mathbf{C}_{4}, \mathbf{C}_{5}$, and $\mathbf{C}_{6}$; and the main index influencing the security criterion $\mathbf{B}_{3}$ is $\mathbf{C}_{7}$. The indexes are defined as follows:

(1) N-1 index $\mathbf{C}_{1}$ : it refers to the total number of elements which does not meet the $N-1$ criterion. The elements that needed $N-1$ check consist of lines, transformers, or main generators.

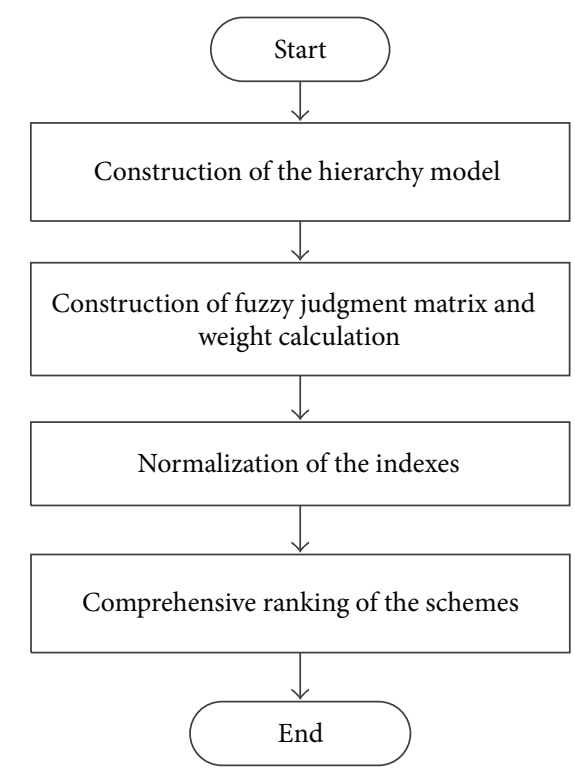

FIGURE 3: Flowchart of the proposed scheme evaluation approach.

(2) The average overload rate of lines index $\mathbf{C}_{2}$ is

$$
\mathbf{C}_{2}=\frac{\sum_{i=1}^{m_{L}} \mathrm{LR}_{i}}{m_{L}}
$$

where $m_{L}$ is the total number of overload lines and $\mathrm{LR}_{i}$ is the load rate of the $i$ th overload line.

(3) The average overload rate of transformers index $\mathbf{C}_{3}$ is

$$
\mathbf{C}_{3}=\frac{\sum_{i=1}^{m_{T}} T_{i}}{m_{T}},
$$

where $m_{T}$ is the total number of overload transformers and $T_{i}$ is the load rate of the $i$ th overload transformer.

(4) The average three-phase short-circuit current index $\mathrm{C}_{4}$ is

$$
\mathbf{C}_{4}=\frac{\sum_{i=1}^{m_{3 P}} \mathrm{SA}_{i}}{m_{3 P}}
$$

where $m_{3 P}$ is the number of buses whose three-phase short-circuit currents exceed the rated value and $\mathrm{SA}_{i}$ is the overcurrent rate of the corresponding bus.

(5) The average single-phase short-circuit current index $\mathrm{C}_{5}$ is

$$
\mathbf{C}_{5}=\frac{\sum_{i=1}^{m_{1 P}} \mathrm{DA}_{i}}{m_{1 P}}
$$

where $m_{1 P}$ is the number of buses whose single-phase short-circuit currents exceed the rated value and $\mathrm{DA}_{i}$ is the overcurrent rate of the corresponding bus. 


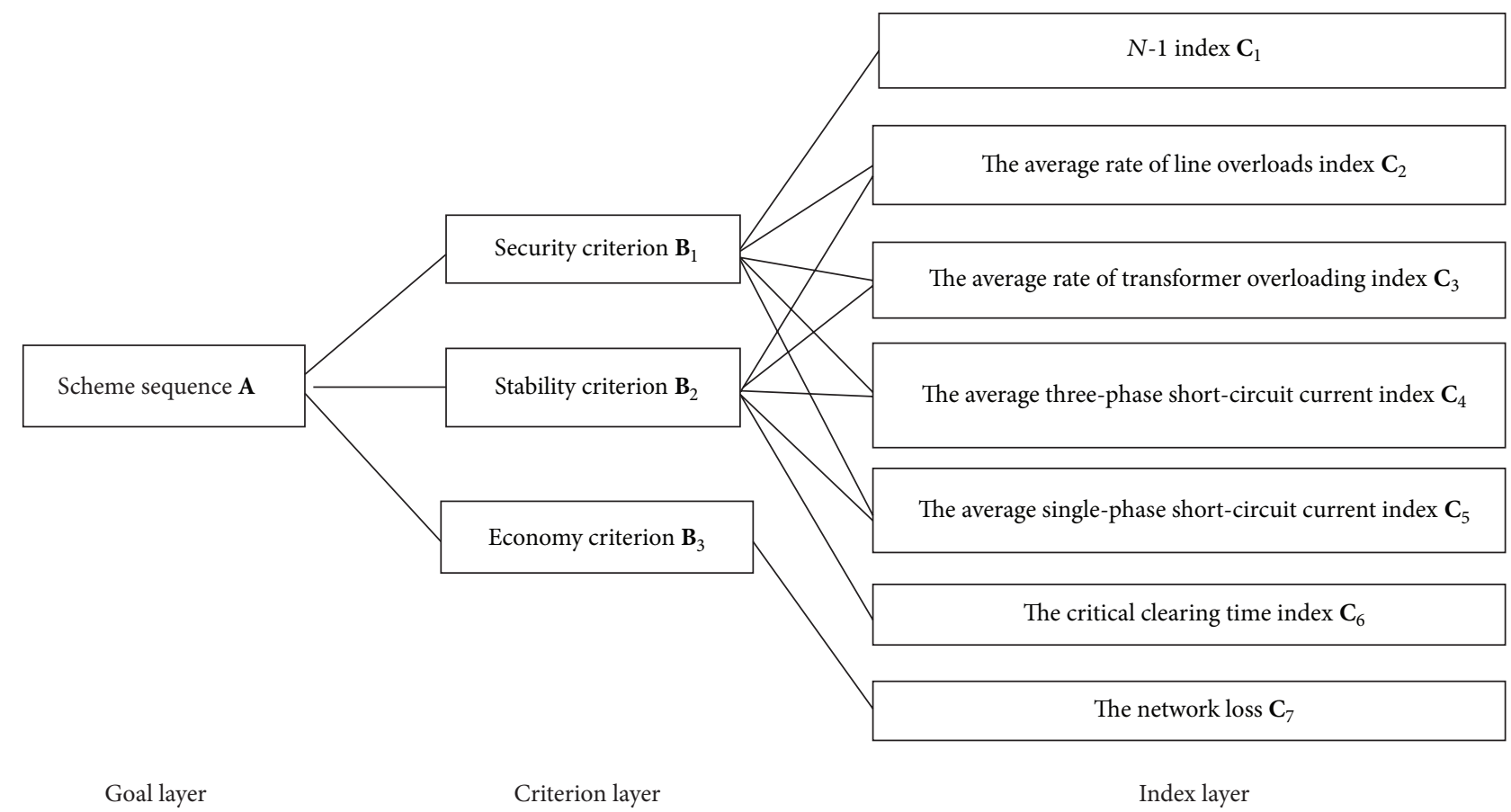

Figure 4: Hierarchical structure model of scheme evaluation.

(6) The critical clearing time index $\mathbf{C}_{6}: \mathbf{C}_{6}$ refers to the critical clearing time when the three-phase shortcircuit fault occurs.

(7) The network loss index $\mathbf{C}_{7}: \mathbf{C}_{7}$ is the active power losses of a power grid.

\subsubsection{Construction of the Fuzzy Judgment Matrix and Weight} Calculation. The complementary judgment matrix using trapezium fuzzy numbers is built according to each layer's relative importance to the top layer. In order to check the judgment matrix's consistency, its kernel matrix is calculated; then, the judgment matrix's consistency is checked through checking its kernel matrix's consistency; after that, fuzzy evaluation of estimate and expectations is calculated, and weight coefficient of each layer is obtained through normalizing the expectations [27].

Step 1. Construction of the initial fuzzy judgment matrix: assuming $L(L \geq 2)$ experts at the equal status are employed simultaneously to build the complementary pairwise comparison judgment matrix in the forms of qualitative evaluations by comparing the importances of all related attributes including $\mathbf{B}_{1} \sim \mathbf{B}_{3}$ in the criterion layer, then, the judgment matrix of the $k$ th expert is described as $\mathbf{R}^{(k)}=$ $\left(r_{i j}^{(k)}\right)_{n \times n}$, where $r_{i j}^{(k)}=\left(a_{i j}^{(k)}, b_{i j}^{(k)}, c_{i j}^{(k)}, d_{i j}^{(k)}\right), k \in\{1,2, \ldots, L\}$; $i, j \in N$.

Step 2. According to the proposed scheme in Section 3.2, check and adjust the consistency of the judgment matrix $\mathbf{R}^{(k)}$ until its consistency is met.
Step 3. Integrate the preference information of the experts according to

$$
\begin{aligned}
r_{i j} & =\left(a_{i j}, b_{i j}, c_{i j}, d_{i j}\right) \\
& =\left(\frac{1}{L} \sum_{k=1}^{L} a_{i j}^{(k)}, \frac{1}{L} \sum_{k=1}^{L} b_{i j}^{(k)}, \frac{1}{L} \sum_{k=1}^{L} c_{i j}^{(k)}, \frac{1}{L} \sum_{k=1}^{L} d_{i j}^{(k)}\right),
\end{aligned}
$$

where the comprehensive fuzzy number complementary judgment matrix $\mathbf{R}=\left(r_{i j}\right)_{n \times n}$.

Step 4. Calculate the weights between the layers. Calculate the fuzzy evaluation value of $y_{i}$ by

$$
\begin{gathered}
v_{i}\left(a_{i}, b_{i}, c_{i}, d_{i}\right)=\left(\frac{\sum_{j=1}^{n} a_{i j}}{\sum_{i=1}^{n} \sum_{j=1}^{n} d_{i j}}, \frac{\sum_{j=1}^{n} b_{i j}}{\sum_{i=1}^{n} \sum_{j=1}^{n} c_{i j}},\right. \\
\left.\frac{\sum_{j=1}^{n} c_{i j}}{\sum_{i=1}^{n} \sum_{j=1}^{n} b_{i j}}, \frac{\sum_{j=1}^{n} d_{i j}}{\sum_{i=1}^{n} \sum_{j=1}^{n} a_{i j}}\right) .
\end{gathered}
$$

Step 5. Supposing that the decision-makers are risk neutral, then calculate the expectation of $y_{i}$ 's fuzzy evaluation value by

$$
I\left(v_{i}\right)=\frac{a_{i}+b_{i}+c_{i}+d_{i}}{4} .
$$

Step 6. According to (11), calculate the weight coefficient of $y_{i}$ by normalizing the expectations to get weight coefficient by

$$
w_{i}=\frac{I\left(v_{i}\right)}{\sum_{i=1}^{n} I\left(v_{i}\right)}, \quad i \in N,
$$

where $w_{i}$ is the weight coefficient of $y_{i}$. 
3.3.3. Normalization of the Indexes. In AHP decision-making method, the index values of all schemes should be normalized. Then, the scheme that has the maximal weight comprehensive evaluation value is the best scheme.

First, $N-1$ index $\left(\mathbf{C}_{1}\right)$ is normalized as

$$
x_{i j}=\frac{\left(1-z_{i j} / N_{\text {threshold }}\right)}{\sum_{i=1}^{m_{s}}\left(1-z_{i j} / N_{\text {threshold }}\right)} \quad(j=1),
$$

where $z_{i j}$ denotes the $\mathbf{C}_{j}$ index value of scheme $S_{i}, x_{i j}$ is the normalized value of the index $\mathbf{C}_{j}$ corresponding to the scheme $S_{i}, m_{S}$ is the total number of alternative schemes to be evaluated, $i \in M, M=\left\{1,2, \ldots, m_{S}\right\}, j \in\{1,2, \ldots, 7\}$, and $N_{\text {threshold }}$ is the threshold value of $N-1$ index, which is predefined based on experts' knowledge. Specifically, if the $N-1$ index of a scheme exceeds the corresponding $N_{\text {threshold }}$, the scheme will not be considered as an alternative one. In this work, $N_{\text {threshold }}$ is set to 10 .

The indexes $\mathbf{C}_{2}, \mathbf{C}_{3}, \mathbf{C}_{4}, \mathbf{C}_{5}$, and $\mathbf{C}_{7}$ are normalized by

$$
x_{i j}=\frac{1 / z_{i j}}{\sum_{i=1}^{m_{S}}\left(1 / z_{i j}\right)} \quad(j=2,3,4,5,7) .
$$

The index $\mathbf{C}_{6}$ is normalized by

$$
x_{i j}=\frac{z_{i j}}{\sum_{i=1}^{m_{s}} z_{i j}} \quad(j=6) \text {. }
$$

3.3.4. Comprehensive Ranking of the Schemes. By the previously mentioned method, the weight vector $W_{A}$ of the criterion layer relative to the goal layer can be obtained. And then, by using the same principle, the weight vector $W_{B}$ of the index layer relative to the criterion layer can also be obtained. The normalized indexes value is represented by $X$, and then the comprehensive evaluation value of each scheme can be obtained: $\mathbf{F}=X * W_{B} * W_{A}$. Finally, the scheme with the maximum comprehensive evaluation value is determined as the optimal scheme.

\section{Case Study}

This section is organized as follows. First, the situation of Liaoning power gird in China is depicted; then, five original schemes to be evaluated are listed; next, the test results using the proposal are shown in detail; finally, comparison tests between the proposal and other evaluation models such as FCE and AHP are carried out, and thereby the corresponding discussions are carried out accordingly.

4.1. Situation of Liaoning Power Gird. The power system considered is the power system of Liaoning province of China consisting of all elements from $10 \mathrm{kV}$ to $500 \mathrm{kV}$. The system covering an area of 148,000 square kilometers is a highly interconnected grid with an approximate installed capacity of 39657.2 MW. The system comprises 91 generators and a total of 750 major buses. The system has some series compensated lines and SVCs. Liaoning Power Electric Network has formed 5 connected channels with the external network through 10
$500 \mathrm{kV}$ AC tie lines, a $1 \pm 500 \mathrm{kV}$ DC line, and $1500 \mathrm{kV}$ DC back-to-back converter station.

In recent years, with the growing strength of grid structures, the north-central grid of Liaoning has formed threedimensional electromagnetic loop networks, which mainly involve voltage grades from $220 \mathrm{kV}$ and $500 \mathrm{kV}$, and the problems of short-circuit current superscalar are serious. Hence, there is an urgent need for decoupling electromagnetic loop networks.

In order to conveniently demonstrate the proposed method, the network diagram of the north-central grid of the power system of Liaoning province is shown in Figure 5.

In Figure 5, the red area represents the existing $500 \mathrm{kV}$ network frame, the green area represents the existing $220 \mathrm{kV}$ network frame, the blue area represents the $220 \mathrm{kV}$ network frame under construction, and the pink area represents the $500 \mathrm{kV}$ network frame under construction.

It can be observed from Figure 5 that the network structure in this area is very dense. With a series of important power transmission and transformation projects comprising Tangjia station and Xihai station, the power supply ability will be greatly increased in this region. But at the same time, the overall short-circuit current level will rise greatly, which may lead to the serious short-circuit current superscalar problem.

4.2. Determination of the Original Schemes. Through large amount of analysis and calculation, five original schemes to be evaluated are listed as follows.

Scheme 1. Disconnect the single loop between Qiangang station and Caohekou station and the single loop between Bohai station and Nanhai station.

Scheme 2. Disconnect the single loop between Bohai station and Nanhai station, the single loop between Bohai station and Xiongyue station, and the double loop between Chengang station and Hongqibao station.

Scheme 3. Disconnect the double loop between Anshan station and Liuerbao station, the double loop between Anshan station and Chengang station, and the double loop between Niuzhuang station and Dongchang station.

Scheme 4. Disconnect the double loop between Anshan station and Wangtie station, the double loop between Anshan station and Chengang station, and the single loop between Qiangang station and Caohekou station.

Scheme 5. Disconnect the single loop between Qiangang station and Caohekou station and the double loop between Anshan station and Liuerbao station.

4.3. Results and Discussion. The actual index values of related schemes are given in Table 2 , where $S_{i}$ denotes the $i$ th scheme.

In Table 2, all the proposed indexes are better if they are smaller except for $\mathbf{C}_{6}$. The indexes have different units, dimensions, and magnitudes, which will affect the decision results, and even cause poor decisions. The normalized index values of related schemes are shown in Table 3. 


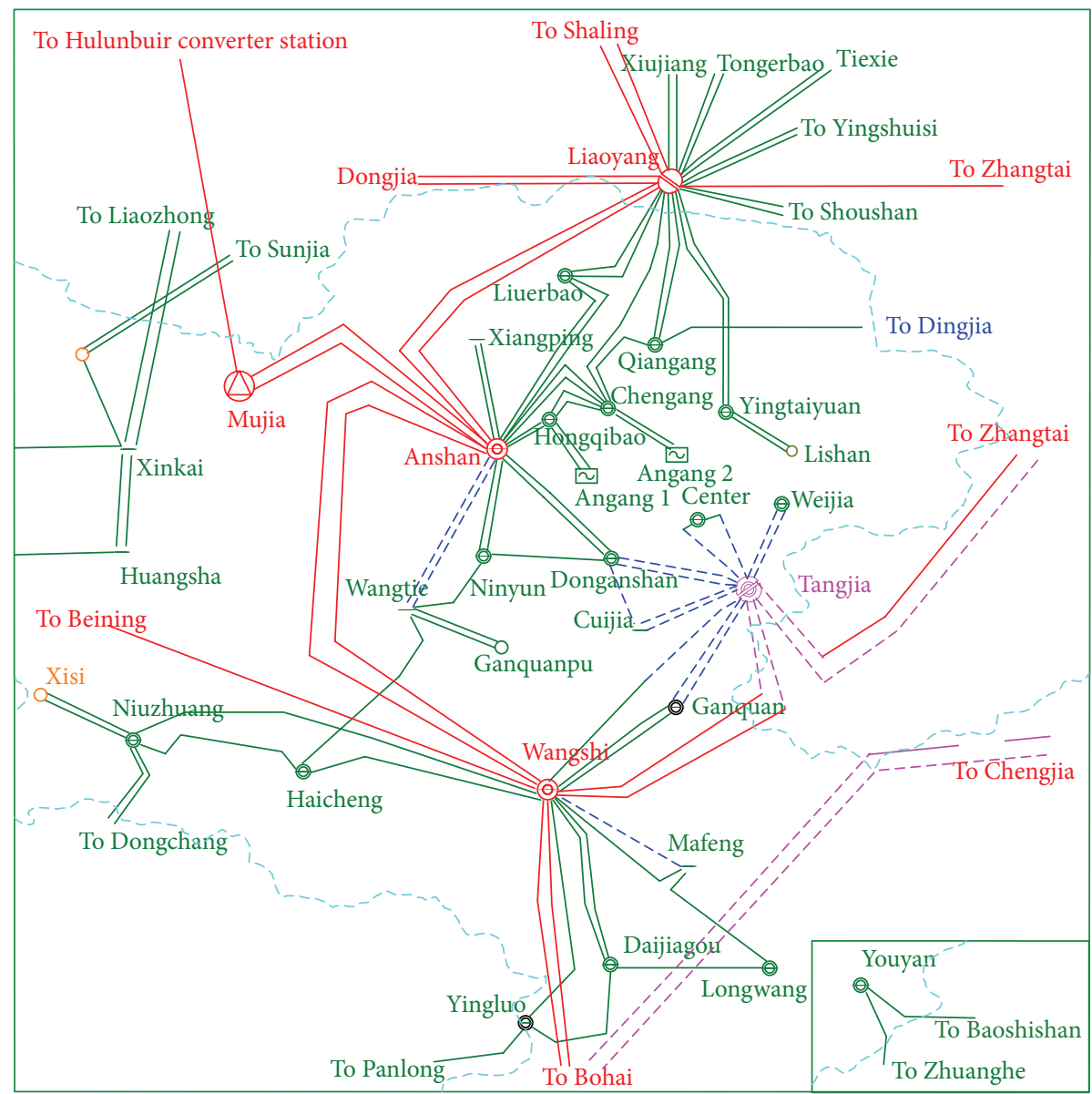

FIGURE 5: Network diagram of the north-central grid of the power system of Liaoning province.

TABLE 2: The actual index values of related schemes.

\begin{tabular}{lccccc}
\hline Index & $S_{1}$ & $S_{2}$ & $S_{3}$ & $S_{4}$ & $S_{5}$ \\
\hline $\mathrm{C}_{1}$ & 5 & 3 & 0 & 2 & 0 \\
$\mathrm{C}_{2}(\%)$ & 93.6 & 84.6 & 86.3 & 82.3 & 82.7 \\
$\mathrm{C}_{3}(\%)$ & 86.7 & 90.1 & 83.5 & 86.7 & 80.8 \\
$\mathrm{C}_{4}$ & 0.063 & 0.061 & 0.062 & 0.064 & 0.066 \\
$\mathrm{C}_{5}$ & 0.178 & 0.167 & 0.141 & 0.158 & 0.161 \\
$\mathrm{C}_{6}(\mathrm{~s})$ & 0.11 & 0.10 & 0.12 & 0.11 & 0.10 \\
$\mathrm{C}_{7}(\mathrm{MW})$ & 295.71 & 235.54 & 241.56 & 308.56 & 286.56 \\
\hline
\end{tabular}

TABLE 3: The normalized index values of related schemes.

\begin{tabular}{lccccc}
\hline Index & $S_{1}$ & $S_{2}$ & $S_{3}$ & $S_{4}$ & $S_{5}$ \\
\hline $\mathrm{C}_{1}$ & 0.125 & 0.175 & 0.250 & 0.200 & 0.250 \\
$\mathrm{C}_{2}(\%)$ & 0.183 & 0.203 & 0.199 & 0.208 & 0.207 \\
$\mathrm{C}_{3}(\%)$ & 0.197 & 0.190 & 0.205 & 0.197 & 0.212 \\
$\mathrm{C}_{4}$ & 0.201 & 0.207 & 0.204 & 0.197 & 0.191 \\
$\mathrm{C}_{5}$ & 0.180 & 0.192 & 0.227 & 0.203 & 0.199 \\
$\mathrm{C}_{6}(\mathrm{~s})$ & 0.204 & 0.185 & 0.222 & 0.204 & 0.185 \\
$\mathrm{C}_{7}(\mathrm{MW})$ & 0.183 & 0.230 & 0.224 & 0.175 & 0.189 \\
\hline
\end{tabular}

Three experts are engaged to build the complementary pairwise comparison judgment matrices by comparing the
TABLE 4: Judgment matrices of criterion layers using qualitative evaluation by the 1st expert.

\begin{tabular}{lccc}
\hline & Security $\mathbf{B}_{1}$ & Stability $\mathbf{B}_{2}$ & Economy $\mathbf{B}_{3}$ \\
\hline Security $\mathbf{B}_{1}$ & $\backslash$ & Generally & Important \\
Stability $\mathbf{B}_{2}$ & Generally & $\backslash$ & Little important \\
Economy $\mathbf{B}_{3}$ & Unimportant & Little unimportant & $\backslash$ \\
\hline
\end{tabular}

TABLE 5: Judgment matrices of criterion layers using qualitative evaluation by the 2 nd expert.

\begin{tabular}{|c|c|c|c|}
\hline & Security $\mathbf{B}_{1}$ & Stability $\mathbf{B}_{2}$ & Economy $\mathbf{B}_{3}$ \\
\hline Security $\mathbf{B}_{1}$ & 1 & Generally & $\begin{array}{c}\text { Very } \\
\text { important }\end{array}$ \\
\hline Stability $\mathbf{B}_{2}$ & Generally & 1 & $\begin{array}{c}\text { Little } \\
\text { important }\end{array}$ \\
\hline Economy $\mathbf{B}_{3}$ & $\begin{array}{c}\text { Very } \\
\text { unimportant }\end{array}$ & $\begin{array}{c}\text { Little } \\
\text { unimportant }\end{array}$ & 1 \\
\hline
\end{tabular}

importances of all related attributes in the criterion layer, and the judgment matrices using qualitative evaluation by the experts are, respectively, shown in Tables $4 \sim 6$. 
TABLE 6: Judgment matrices of criterion layers using qualitative evaluation by the 3 rd expert.

\begin{tabular}{lccc}
\hline & Security $\mathbf{B}_{1}$ & Stability $\mathbf{B}_{2}$ & Economy $\mathbf{B}_{3}$ \\
\hline Security $\mathbf{B}_{1}$ & $\backslash$ & Generally & Very important \\
Stability $\mathbf{B}_{2}$ & Generally & $\backslash$ & Important \\
Economy $\mathbf{B}_{3}$ & Very unimportant & Unimportant & $\backslash$ \\
\hline
\end{tabular}

And then, based on the judgment matrices using qualitative evaluation, the trapezoidal fuzzy number complementary judgment matrices $\mathbf{R}^{(1)} \sim \mathbf{R}^{(3)}$ are obtained according to Table 1:

$$
\begin{aligned}
& \mathbf{R}^{(1)} \\
& =\left[\begin{array}{lll}
{[0.5,0.5,0.5,0.5]} & {[0.3,0.5,0.5,0.7]} & {[0.7,0.9,1.0,1.0]} \\
{[0.3,0.5,0.5,0.7]} & {[0.5,0.5,0.5,0.5]} & {[0.6,0.8,0.8,1.0]} \\
{[0.0,0.0,0.1,0.3]} & {[0.0,0.2,0.2,0.4]} & {[0.5,0.5,0.5,0.5]}
\end{array}\right],
\end{aligned}
$$

$\mathbf{R}^{(2)}$

$=\left[\begin{array}{lll}{[0.5,0.5,0.5,0.5]} & {[0.3,0.5,0.5,0.7]} & {[0.8,1.0,1.0,1.0]} \\ {[0.3,0.5,0.5,0.7]} & {[0.5,0.5,0.5,0.5]} & {[0.6,0.8,0.8,1.0]} \\ {[0.0,0.0,0.0,0.2]} & {[0.0,0.2,0.2,0.4]} & {[0.5,0.5,0.5,0.5]}\end{array}\right]$,

$\mathbf{R}^{(3)}$

$$
=\left[\begin{array}{lll}
{[0.5,0.5,0.5,0.5]} & {[0.3,0.5,0.5,0.7]} & {[0.8,1.0,1.0,1.0]} \\
{[0.3,0.5,0.5,0.7]} & {[0.5,0.5,0.5,0.5]} & {[0.7,0.9,1.0,1.0]} \\
{[0.0,0.0,0.0,0.2]} & {[0.0,0.0,0.1,0.3]} & {[0.5,0.5,0.5,0.5]}
\end{array}\right] .
$$

The consistency indexes of the complementary judgment matrices are $\rho_{1}=0.0917, \rho_{2}=0.1333$, and $\rho_{3}=0.0417$, respectively. Note that all the consistency indexes meet the requirements of consistency since they are all smaller than the threshold 0.2. By using the weighted average expert advice, the trapezoidal fuzzy judgment matrix $\mathbf{R}$ of the criterion layer relative to the goal layer is

$$
\mathbf{R}=\left[\begin{array}{cccc}
(0.50,0.50,0.50,0.50) & (0.30,0.50,0.50,0.70) & (0.77,0.97,1.00,1.00) \\
(0.30,0.50,0.50,0.70) & (0.50,0.50,0.50,0.50) & (0.63,0.83,0.87,1.00) \\
(0.00,0.00,0.03,0.23) & (0.00,0.13,0.17,0.37) & (0.50,0.50,0.50,0.50)
\end{array}\right]
$$

The weight vector of the criterion layers is $W_{A}=$ $\left[\begin{array}{lll}0.4009 & 0.4168 & 0.1824\end{array}\right]^{T}$.

By using the same method, the weight vector of the index layer of $\mathbf{C}_{1}, \mathbf{C}_{2}, \mathbf{C}_{3}, \mathbf{C}_{4}$, and $\mathbf{C}_{5}$ relative to the criterion layer $\mathbf{B}_{1}$ is $W_{B 1}=\left[\begin{array}{lllllll}0.250 & 0.034 & 0.066 & 0.350 & 0.267 & 0 & 0\end{array}\right]^{T}$; the weight vector of the index layer of $\mathbf{C}_{1}, \mathbf{C}_{2}, \mathbf{C}_{3}, \mathbf{C}_{4}$, $\mathbf{C}_{5}$, and $\mathbf{C}_{6}$ relative to the criterion layer $\mathbf{B}_{2}$ is $W_{B 2}=$

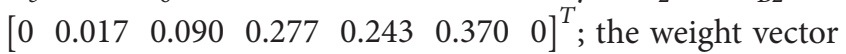
of the index layer of $\mathrm{C}_{7}$ relative to the criterion layer $B_{3}$ is $W_{B 3}=\left[\begin{array}{lllllll}0 & 0 & 0 & 0 & 0 & 0 & 1\end{array}\right]^{T}$. And then, the weight vector $W_{B}$ of the index layer relative to the criterion layer can be obtained:

$$
W_{B}=\left[W_{B 1}, W_{B 2}, W_{B 3}\right]=\left[\begin{array}{ccc}
0.250 & 0 & 0 \\
0.034 & 0.017 & 0 \\
0.066 & 0.090 & 0 \\
0.350 & 0.277 & 0 \\
0.267 & 0.243 & 0 \\
0 & 0.370 & 0 \\
0 & 0 & 1
\end{array}\right] \text {. }
$$

The comprehensive evaluation values of the candidate schemes are $\mathbf{F}=X * W_{B} * W_{A}=[0.1824,0.1972$, $0.2167,0.1930,0.1962]^{T}$. Consequently, the comprehensive ranking of the schemes is $S_{3}>S_{2}>S_{5}>S_{4}>S_{1}$. Therefore, the conclusion can be drawn that $S_{3}$ is the optimal scheme, and $S_{1}$ is the worst scheme.

From the above results, the scheme $S_{3}$ has the best values of $\mathbf{C}_{1}, \mathbf{C}_{5}$, and $\mathbf{C}_{6}$; meanwhile, all the other indexes of the scheme are pretty good. On the contrary, the scheme $S_{1}$ has the worst values of $\mathbf{C}_{1}, \mathbf{C}_{2}$, and $\mathbf{C}_{5}$; all the other indexes of the scheme are almost the worst. Consequently, the scheme $S_{3}$ is suitable to be used as the final opening scheme in this work, which can effectively give consideration to all factors relating to the problem of determination of optimal opening scheme.

4.4. Test Results of Other Evaluation Models. In order to further verify the superiority of the proposed method to the traditional AHP method, comparison tests are, respectively, carried out by using FCE [12] and AHP [9, 11], and the results comprising priorities and sequences are shown in Table 7.

From the results in Table 7, it can be seen that the ranking results by using the proposed method and FCE are consistent. Specifically, the scheme $S_{3}$ is the optimal decoupling scheme and the scheme $S_{1}$ is the worst one. This evaluation result is also in line with the actual analysis and judgments of the power system planning and design personnel, which demonstrates that the proposed approach is feasible and has a certain practical value.

At the same time, Table 7 also shows the result using AHP is different from the proposed method. More specifically, the scheme $S_{2}$ is superior to $S_{3}$ when using AHP, while the result is just the opposite when using the proposal. The reason 
TABLE 7: The evaluation results of other related schemes.

\begin{tabular}{llccccc}
\hline \multirow{2}{*}{ Evaluation model } & $S_{1}$ & $S_{2}$ & $S_{3}$ & $S_{4}$ & $S_{5}$ \\
\multirow{2}{*}{ FCE } & Priority & 0.286 & 0.316 & 0.326 & 0.296 & 0.309 \\
& Sequence & 5 & 2 & 1 & 4 & 3 \\
\hline \multirow{2}{*}{ AHP } & Priority & 0.199 & 0.239 & 0.229 & 0.205 & 0.219 \\
& Sequence & 5 & 1 & 2 & 4 & 3 \\
\hline \multirow{2}{*}{ IFAHP } & Priority & 0.182 & 0.197 & 0.217 & 0.193 & 0.196 \\
& Sequence & 5 & 2 & 1 & 4 & 3 \\
\hline
\end{tabular}

for this is that there is strong subjectivity when using AHPbased decision-making model, which needs experts with rich experience to pairwise compare the importances of all related attributes in the criterion layer when building the judgment matrices. If there are many indexes to be compared like the case in this paper while at the same time the engaged experts' knowledge is inadequate, the judgment bias problem will inevitably occur. It is just because of this reason that the ranking results of the proposed approach and AHP are different. Therefore, the conclusion can be drawn on the basis of the evidence that, compared with the conventional AHP, the ranking results by using the proposal are more scientific, reasonable, and objective, and thereby the superiority of the proposal is verified.

\section{Conclusions and Future Research}

In this paper, an IFAHP-based MCDM method is proposed for determination of optimal opening scheme for electromagnetic loop networks. The proposed approach is examined on a real power system - the power system of Liaoning province. The main lessons learnt from the proposed method are as follows:

(1) The proposed approach combines the advantages of AHP and fuzzy theory, and it effectively overcomes the limitations of AHP when dealing with subjective factors of different experts.

(2) The presented method can comprehensively, objectively, and scientifically evaluate the pros and cons of different schemes and provides an important reference for the planning and construction of power grids.

(3) The effectiveness of the proposed method is validated by the application results on the real power system. Furthermore, it may be applied to similar MCDM problems in engineering field.

The contribution of this paper can be divided into two aspects: on the one hand, how IFAHP, a new MCDM method, is improved and applied to solve the problem of determination of optimal opening scheme for electromagnetic loop networks in detail is demonstrated; on the other hand, significant performance improvements from applying the present approach to test systems are further demonstrated.

Compared to other MCDM methods, the proposed approach can combine the advantages of AHP and fuzzy set theory. On the one hand, AHP guarantees the systematicness and rationality of the decision-making model by combining qualitative analysis with quantitative analysis effectively; on the other hand, due to fully considering the fuzzy nature of experts' knowledge, fuzzy set theory contributes to ensure the objectivity of experts' knowledge by representing the elements of the judgment matrix in the form of a linguistic term set. In addition, compared to FAHP, it overcomes the inherent defects of FAHP in checking and adjusting the consistency of the fuzzy judgment matrix.

In future research, one interesting direction might be considering other state-of-the-art MCDM methods such as ELECTRE [28], TOPSIS [29], and BWM [30]. In addition, the case discussed in this paper assumes that the original opening schemes are obtained through large amount of analysis and calculation, which is time-consuming and largely dependent on experts' experience. Consequently, another direction might be developing programs to generate the original opening schemes automatically, which will greatly improve the practicability of the proposed method.

\section{Nomenclature}

\begin{tabular}{|c|c|}
\hline MCDM: & Multicriteria decision-making \\
\hline FCE: & Fuzzy comprehensive evaluation \\
\hline AHP: & Analytic hierarchy process \\
\hline FAHP: & Fuzzy AHP \\
\hline IFAHP: & Improved FAHP \\
\hline SCC: & Short-circuit current \\
\hline VSC-HVDC: & $\begin{array}{l}\text { Voltage source converter-high voltage } \\
\text { direct current }\end{array}$ \\
\hline ELECTRE: & $\begin{array}{l}\text { Elimination and Choice Expressing } \\
\text { Reality }\end{array}$ \\
\hline TOPSIS: & $\begin{array}{l}\text { Technique for Order Preference by } \\
\text { Similarity to an Ideal Solution }\end{array}$ \\
\hline BWM & Best Worst Method. \\
\hline
\end{tabular}

\section{Competing Interests}

The authors declare that they have no competing interests.

\section{Acknowledgments}

This research is supported by the Science and Technology Project of State Grid Corporation of China under Grant no. 2014GW-05, the key technology research of a flexible ring network controller and its demonstration application.

\section{References}

[1] D. Yang, K. Zhao, and Y. Liu, "Optimization and decision for opening electromagnetic loop circuits in ultra-high voltage grid," High Voltage Engineering, vol. 41, no. 3, pp. 778-786, 2015.

[2] M. S. Liu, J. Zhang, and W. C. Zhang, "Problems and countermeasures for operation and control of weak electromagnetic loops," Automation of Electric Power Systems, vol. 38, no. 4, pp. 109-114, 2014.

[3] D. Yang and Y. Liu, "Influence of electromagnetic loop in early ultra-high voltage grid," Electric Power Automation Equipment, vol. 29, no. 6, pp. 77-84, 2009. 
[4] J. Liu, C. Yi, J. Zhao, Q. Zhou, and Q. Li, "Study on the method of evaluating open/closed loop operation modes of electromagnetic loop network based on group decision-making theory," in Proceedings of the IEEE International Conference on Electricity Distribution (CICED '14), pp. 1440-1445, Shenzhen, China, September 2014.

[5] K. Gao, Y. Y. Yang, Y. J. Zhang, Q. Li, and Z. Han, "A topology research of flexible looped network controller suitable to urban power grid," Power System Technology, vol. 40, no. 1, pp. 78-85, 2016.

[6] A. Marinakis, M. Glavic, and T. Van Cutsem, "Minimal reduction of unscheduled flows for security restoration: application to phase shifter control," IEEE Transactions on Power Systems, vol. 25, no. 1, pp. 506-515, 2010.

[7] G. P. Granelli, M. Montagna, F. Zanellini, P. Bresesti, and R. Vailati, "A genetic algorithm-based procedure to optimize system topology against parallel flows," IEEE Transactions on Power Systems, vol. 21, no. 1, pp. 333-340, 2006.

[8] H. Ye, Y.-T. Liu, and X.-S. Niu, "Fuzzy comprehensive evaluation of opening schemes for $500 \mathrm{kV}-220 \mathrm{kV}$ electromagnetic loop," Electric Power Automation Equipment, vol. 26, no. 7, pp. 1-5, 2006.

[9] N. Zhang, H. Ye, and Y. T. Liu, "Decision support for choosing optimal electromagnetic loop circuit opening scheme based on analytic hierarchy process and multi-level fuzzy comprehensive evaluation," Engineering Intelligent Systems for Electrical Engineering and Communications, vol. 16, no. 4, pp. 183-191, 2008.

[10] J. Rezaei, P. B. M. Fahim, and L. Tavasszy, "Supplier selection in the airline retail industry using a funnel methodology: conjunctive screening method and fuzzy AHP," Expert Systems with Applications, vol. 41, no. 18, pp. 8165-8179, 2014.

[11] W. Pedrycz and M. Song, "Analytic Hierarchy process (AHP) in group decision making and its optimization with an allocation of information granularity," IEEE Transactions on Fuzzy Systems, vol. 19, no. 3, pp. 527-539, 2011.

[12] Y. Liu, P. Fang, D. Bian, H. Zhang, and S. Wang, "Fuzzy comprehensive evaluation for the motion performance of autonomous underwater vehicles," Ocean Engineering, vol. 88, pp. 568-577, 2014.

[13] T. Chen, Y. Jin, X. Qiu, and X. Chen, "A hybrid fuzzy evaluation method for safety assessment of food-waste feed based on entropy and the analytic hierarchy process methods," Expert Systems with Applications, vol. 41, no. 16, pp. 7328-7337, 2014.

[14] L. A. Zadeh, "Fuzzy sets," Information and Computation, vol. 8, pp. 338-353, 1965.

[15] C. Kahraman, S. C. Onar, and B. Oztaysi, "Fuzzy multicriteria decision-making: a literature review," International Journal of Computational Intelligence Systems, vol. 8, no. 4, pp. 637-666, 2015.

[16] K. K. F. Yuen, "Fuzzy cognitive network process: comparisons with fuzzy analytic hierarchy process in new product development strategy," IEEE Transactions on Fuzzy Systems, vol. 22, no. 3, pp. 597-610, 2014.

[17] G. van de Kaa, E. van Heck, H. J. de Vries, J. van den Ende, and J. Rezaei, "Supporting decision making in technology standards battles based on a fuzzy analytic hierarchy process," IEEE Transactions on Engineering Management, vol. 61, no. 2, pp. 336348, 2014.

[18] T. N. Le, H. A. Quyen, and N. A. Nguyen, "Application of fuzzyanalytic hierarchy process algorithm and fuzzy load profile for load shedding in power systems," International Journal of Electrical Power and Energy Systems, vol. 77, pp. 178-184, 2016.
[19] L. Mikhailov, "Deriving priorities from fuzzy pairwise comparison judgements," Fuzzy Sets and Systems, vol. 134, no. 3, pp. 365385, 2003.

[20] J. Rezaei, R. Ortt, and V. Scholten, "An improved fuzzy preference programming to evaluate entrepreneurship orientation," Applied Soft Computing Journal, vol. 13, no. 5, pp. 2749-2758, 2013.

[21] Y.-M. Wang, J.-B. Yang, and D.-L. Xu, "A two-stage logarithmic goal programming method for generating weights from interval comparison matrices," Fuzzy Sets and Systems, vol. 152, no. 3, pp. 475-498, 2005.

[22] S. Cho, J. Kim, and E. Heo, "Application of fuzzy analytic hierarchy process to select the optimal heating facility for Korean horticulture and stockbreeding sectors," Renewable and Sustainable Energy Reviews, vol. 49, pp. 1075-1083, 2015.

[23] L. A. Zadeh, "The concept of a linguistic variable and its applications to approximate reasoning," Information Sciences, Part I, II, III, vol. 8, pp. 199-249, 1975.

[24] K. R. MacCrimmon and D. A. Wehrung, "Characteristics of risk taking executives," Management Science, vol. 36, no. 4, pp. 422435, 1990.

[25] K. Kułakowski, "Notes on order preservation and consistency in AHP," European Journal of Operational Research, vol. 245, no. 1, pp. 333-337, 2015.

[26] G. X. Song and D. L. Yang, "Methods for identifying and improving the consistency of fuzzy judgment matrix," Systems Engineering, vol. 21, no. 1, pp. 110-116, 2003.

[27] C. A. Chen and H. L. Lee, "The study of how to improve the consistency of the AHP and ANP methods," Journal of Data Analysis, vol. 10, no. 3, pp. 115-140, 2015.

[28] K. Govindan and M. B. Jepsen, "ELECTRE: a comprehensive literature review on methodologies and applications," European Journal of Operational Research, vol. 250, no. 1, pp. 1-29, 2016.

[29] Ü. Şengül, M. Eren, S. E. Shiraz, V. Gezder, and A. B. Şengül, "Fuzzy TOPSIS method for ranking renewable energy supply systems in Turkey," Renewable Energy, vol. 75, pp. 617-625, 2015.

[30] J. Rezaei, "Best-worst multi-criteria decision-making method," Omega, vol. 53, pp. 49-57, 2015. 


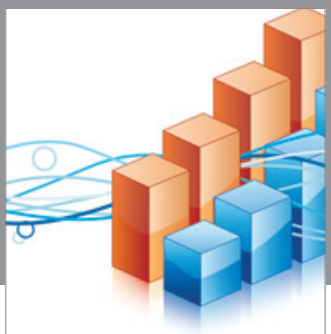

Advances in

Operations Research

vatem alat4

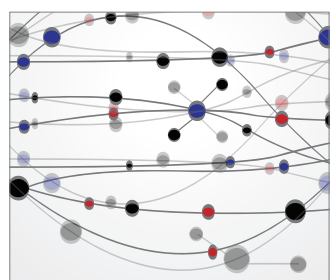

\section{The Scientific} World Journal
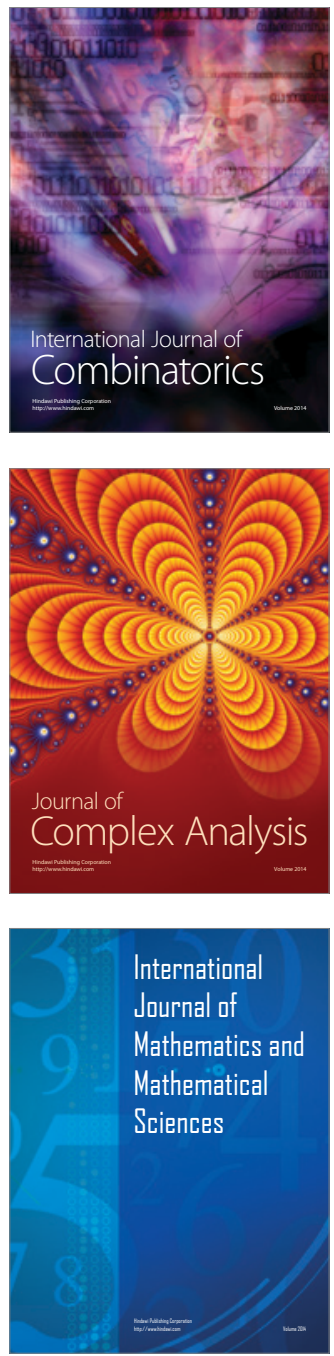
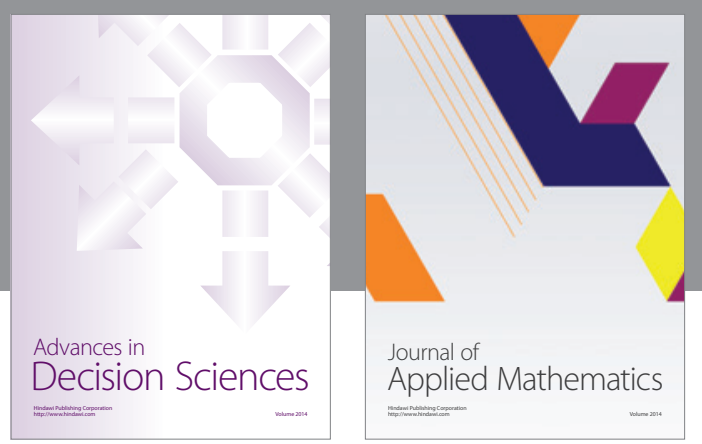

Algebra

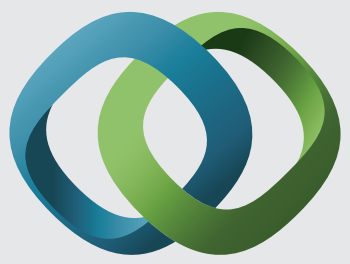

\section{Hindawi}

Submit your manuscripts at

http://www.hindawi.com
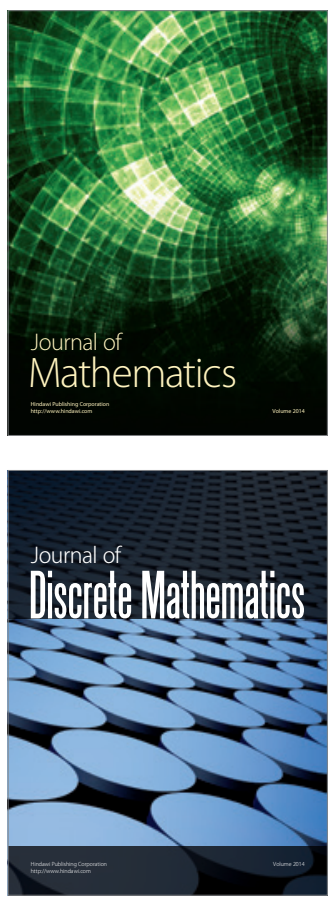

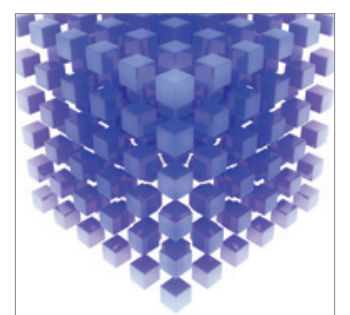

Mathematical Problems in Engineering
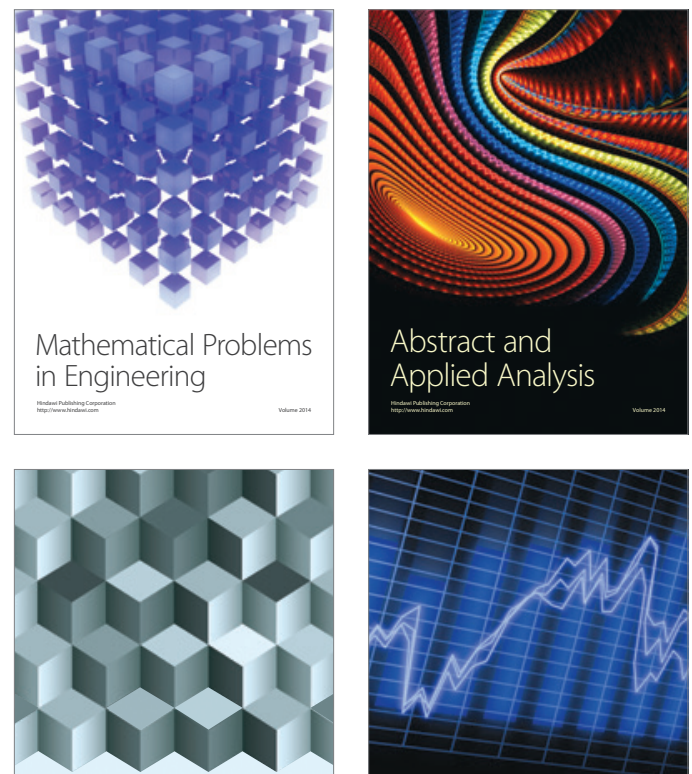

Journal of

Function Spaces

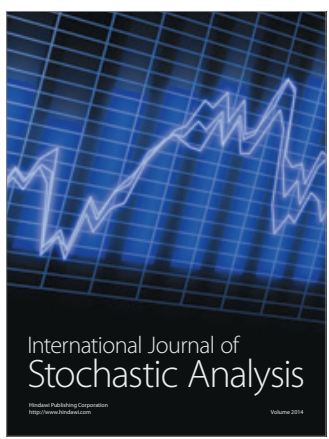

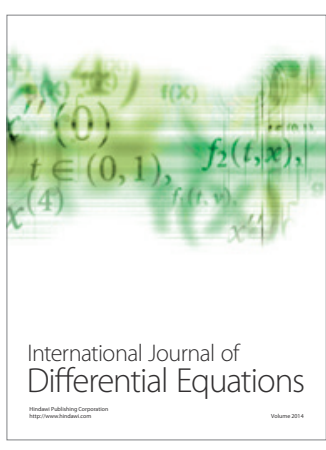
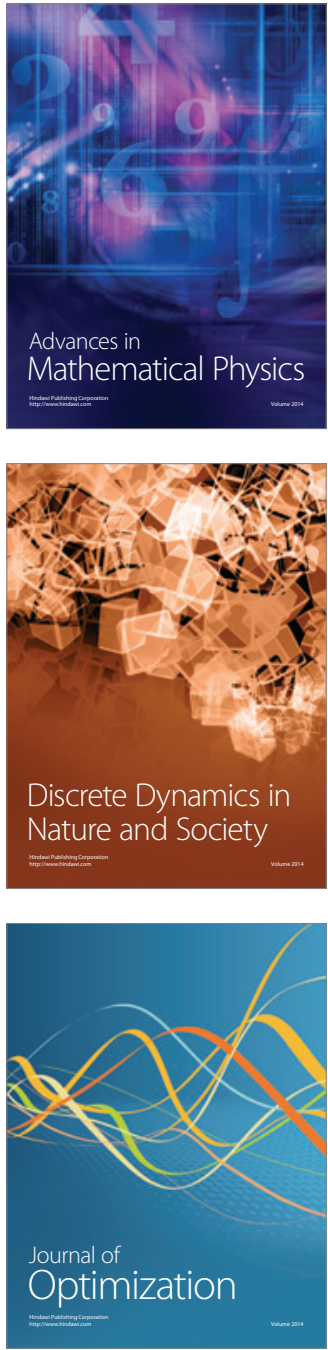